\title{
Design on Parallel Structure Multi-frequency Fx-LMS Calculation of Signal Processing Filter with Errors
}

\author{
ZHOU Lujun ${ }^{1, a}$, ZHOU Daoqing ${ }^{2, b}$, NIE Yongming ${ }^{3, c}$, RU Hailong ${ }^{4, d}$, HAO \\ Yong ${ }^{5, \mathrm{e}}$
}

China Satellite Maritime Tracking and Controlling Department, Jiangyin, 214431, China

aemail:zhoulujun168@163.com, bemail:623943019@qq.com, email:yimonie@163.com

Keywords: Adaptive filtering, Parallel structure, ACSR, Multi-frequency Fx-LMS calculation

Abstract. On the basis of introducing Adaptive Filter Theory, this essay analyzes the stability and inductive spectrum of traditional parallel structure Fx-LMS calculation and carries out a modified parallel structure multi-frequency Fx-LMS calculation of signal processing filter with errors. Damping vibration control calculation aiming at helicopter $k \Omega$ and $2 k \Omega$ 's vibration frequency components is designed so that the stability and control effect of Active Control Structure Response (ACSR) system was improved.

\section{Introduction}

ACSR is a high and new technology which attracts much attention at home and abroad in helicopter engineering industry nowadays and self-adaptive control is one of the cores of this technology. The traditional parallel structure time domain Fx-LMS multi-frequency calculation is simple and efficient, but also some problems exist in stability and induction spectrum. Towards this situation, this essay comes up with a modified parallel structure multi-frequency calculation of signal processing filter with errors and designed a damping vibration calculation for $k \Omega$ and $2 k \Omega$ 's vibration frequency component after considering the actual vibration damping demand, which can improve stability and control effect of ACSR system[1].

\section{The Fundamental Principle of Adaptive Filtering}

Adaptive filter is a digital filter that can deal with digital signals in accordance with output signal automatic adjustment function. It consists of two parts: digital filter and adaptive calculation. The fundamental working principle is: adaptive calculation adjusts the parameter and structure according to the changes of outside environment so that the output of filter can reach the expected response signal. No need for accurate model of the control object and simple calculation for achieving real-time control system are the advantages of this calculation[2-4]. The principle chart of adaptive filter is shown in Figure 1.

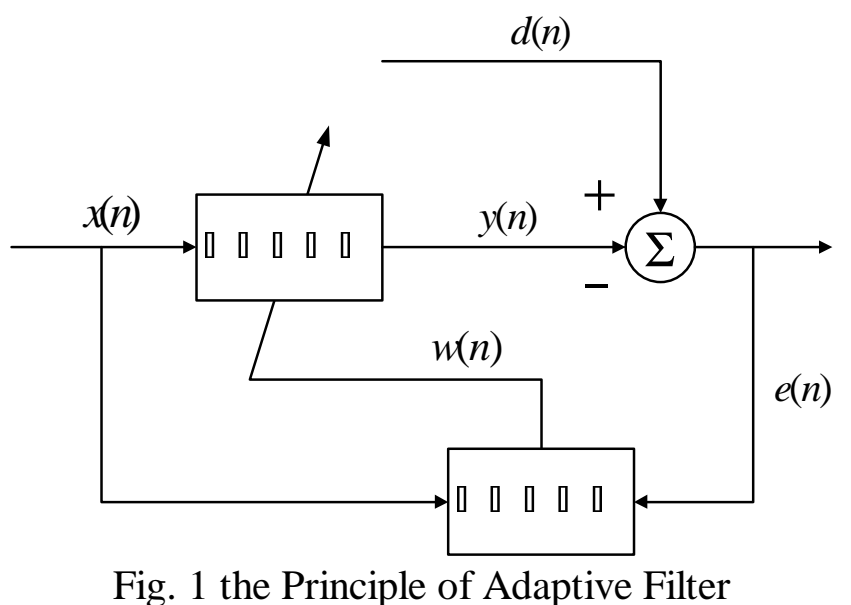

Fig. 1 the Principle of Adaptive Filter 
In Figure 1, $d(n)$ is desired signal and is also vibration signal which is needed to be controlled in controlled point; $x(n)$ is reference signal that is related to rotor wing external disturbance simulation from helicopter rotor wing rotation speed signal; $x(n)^{\prime} \mathrm{s}$ output signal is $y(n)$ by getting through digital filter; $e(n)$ is the superposition of surplus error signals after offsetting $d(n)$ and $y(n)$ and response external disturbance simulation as well as vibration signal generated in controlled point by actuator. The function of adaptive calculation is adjusting the parameter of adaptive filter constantly on the basis of particular calculation so that the mean square value $e(n)$ of error signal from system output will be minimized.

\section{Analysis of Parallel Structure Multi-frequency Fx-LMS Calculation}

In actual situation, periodic vibration which caused by rotation system such as engine consisted of many narrow-band frequency components like main passing frequency and high-order harmonic wave. This kind of active control system was usually applied by parallel structure multi-frequency Fx-LMS calculation[5-6]. In case that desired signal includes $M$ independent sine components, these sine signals can be controlled by $\mathrm{M}$ independent adaptive filter respectively, which is shown in Figure 2.

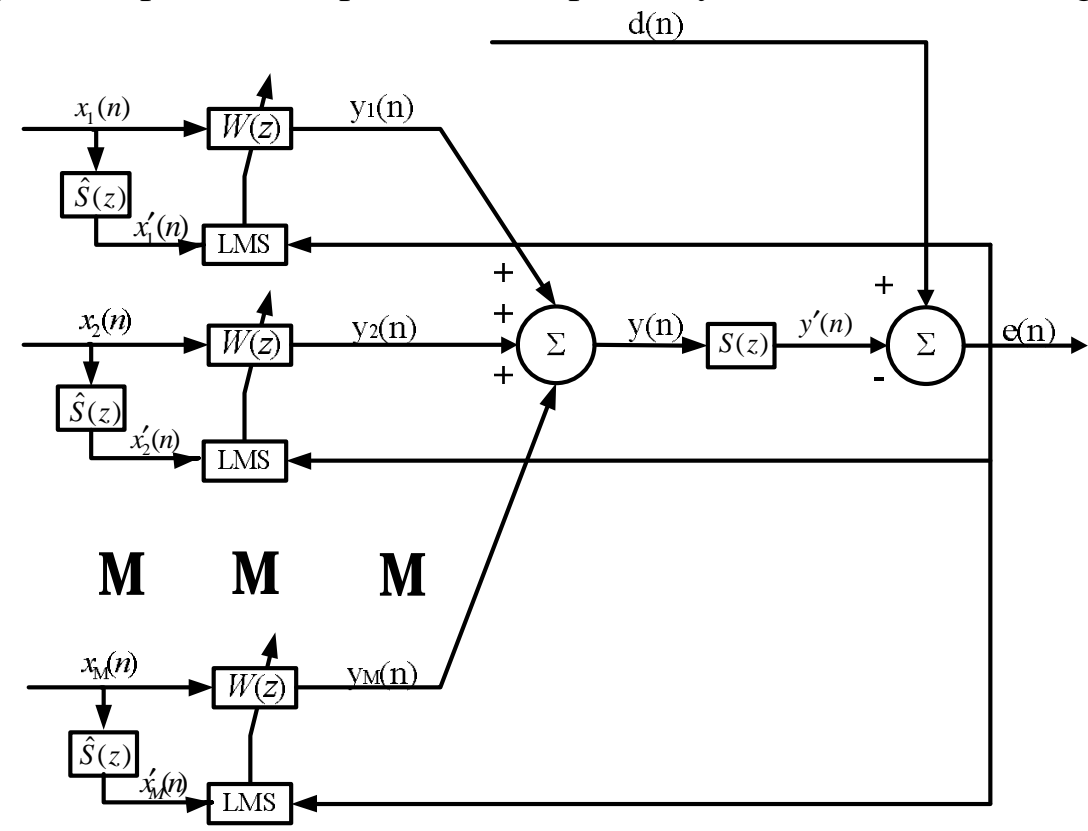

Fig. 2 Structure Diagram of Parallel Structure Multi-frequency Narrow-band Control

In this figure, $\hat{S}(z)$ represents $S(z)^{\prime}$ s mathematic model.

\section{(1)The Stability Analysis of Parallel Structure Multi-frequency Fx-LMS Calculation}

As is shown in Figure 2, desired signal includes M sine frequency spectrums:

$$
d(n)=\sum_{m=1}^{M} A_{d, m} \sin \left(\omega_{m} n+\phi_{d, m}\right)
$$

In this formula, $A_{d, m}$ and $\phi_{d, m}$ are the amplitudes and phases of sine signal whose frequency is $\omega_{m}$. And the No.m control path' s reference signal can be shown as:

$$
x_{m}(n)=A_{d, m} \sin \left(\omega_{m} n\right), \quad m=1,2, \ldots, M
$$

The sum of all filter output can be shown as:

$$
y(n)=\sum_{m=1}^{M} y_{m}(n), \quad m=1,2, \ldots, M
$$


In this formula,,$y_{m}(n)$ is ACSR' s output of No.m path.

The autocorrelation matrix of input signal is :

In this formula, the sub matrixes are:

$$
R=\left[\begin{array}{rrrr}
R_{11} & R_{12} & \mathrm{~L} & R_{1 M} \\
R_{21} & R_{22} & \mathrm{~L} & R_{2 M} \\
\mathrm{M} & \mathrm{M} & \mathrm{O} & \mathrm{M} \\
R_{M 1} & R_{M 2} & \mathrm{~L} & R_{M M}
\end{array}\right]
$$

$$
R_{p q}=E\left[x_{p}^{\prime}(n) x_{q}^{\prime T}(n)\right], \quad p, q=1,2, \ldots, M
$$

This formula shows the autocorrelation matrix of paths No.p and No.q' s reference signal (sine signal). If using a control system which is similar to single frequency vibrations control system[7], then:

as well as

$$
R_{p q}=0_{2 \times 2}, \quad p \neq q \text { 且 } p, q=1,2, \ldots, M
$$

$$
R_{p p}=\frac{A_{P}^{\prime 2}}{2}\left[\begin{array}{cc}
1 & \cos \omega_{p} \\
\cos \omega_{p} & 1
\end{array}\right], \quad p=1,2, \ldots, M
$$

In this formula, $A_{P}^{\prime}$ is the product of reference signal amplitude $A_{P}$ and the No.p secondary path amplitude response $A_{S, P}$, that is $A_{P}^{\prime}=A_{P} A_{S, P}$. So formula (4) can be shown as:

$$
R=\left[\begin{array}{llll}
R_{11} & & & \\
& R_{22} & & \\
& & \mathrm{O} & \\
& & & R_{M M}
\end{array}\right]=\Lambda R_{p p}, p=1,2, \mathrm{~K}, M
$$

$\Lambda$ means opposite angle in the formula.

And cross-correlation $\mathrm{p}$ is:

And sub matrixes are:

$$
p=\left[\begin{array}{lll}
p_{1}^{T} & p_{2}^{T} \mathrm{~L} & p_{M}^{T}
\end{array}\right]^{T}
$$

$$
p_{p}=E\left\lfloor d\left(n x_{p}^{\prime}(n)\right)\right\rfloor, \quad p=1,2, \mathrm{~K}, M
$$

These sub matrixes show the cross-correlation matrix of desired signal and reference signal of No.p path, then:

$$
p_{p}=\frac{A_{P}^{\prime} A_{d, p}}{2}\left[\begin{array}{c}
\cos \left(\phi_{d, p}-\phi_{s, p}\right) \\
\cos \left(\omega_{p}+\phi_{d, p}-\phi_{s, p}\right)
\end{array}\right], \quad p=1,2, \mathrm{~K}, M
$$

In this formula, $\phi_{s, p}$ represents phase response of the No.p secondary path.

To sum up, the optimal weight vector matrix is:

and:

$$
w^{o}=\left[\left(w_{1}^{o}\right)^{T}\left(w_{2}^{o}\right)^{T} \mathrm{~L}\left(w_{M}^{o}\right)^{T}\right]^{T}
$$

$$
w_{p}^{o}=R_{p p}^{-1} p_{p}=\frac{A_{d, p}}{A_{p}^{\prime} \sin \omega_{p}}\left[\begin{array}{c}
\sin \left(\omega_{p}+\phi_{d, p}-\phi_{s, p}\right) \\
-\sin \left(\phi_{d, p}-\phi_{s, p}\right)
\end{array}\right], \quad p=1,2, \mathrm{~K}, M
$$

The proper value of every sub matrix $R_{P P}$ is:

$$
\lambda_{l, p}=\frac{A_{P}^{\prime 2}}{2}\left(1 \pm \cos \omega_{p}\right), \quad l=0,1 \text { 和 } p=1,2, \mathrm{~K}, M
$$

To assume the maximum and minimum of these proper values:

$$
\lambda_{\max }=\frac{A_{k}^{\prime 2}}{2}\left(1+\left|\cos \omega_{k}\right|\right)
$$




$$
\lambda_{\min }=\frac{A_{h}^{\prime 2}}{2}\left(1-\left|\cos \omega_{h}\right|\right)
$$

The proper value diffusivity of the whole control system which concluded from the definition of proper value diffusivity in reference [8]:

$$
\rho=\frac{\lambda_{\max }}{\lambda_{\min }}=\frac{A_{k}^{\prime 2}\left(1-\left|\cos \omega_{k}\right|\right)}{A_{h}^{\prime 2}\left(1-\left|\cos \omega_{h}\right|\right)}
$$

The formula above shows that secondary path transfer function influences the stability of the whole control system.

\section{(2)Induction Component Analysis of Parallel Structure Multi-frequency Fx-LMS Calculation}

According to reference [9], when error signal has many spectrums, other harmonic wave components can be induced, which may influence the efficiency of calculation control. Analysis following will be the reasons for generating harmonic components when taking three frequency spectrums ' error signal as examples.

$$
\begin{aligned}
& \text { When } \mathrm{k}=1 \text {, } \\
& \mu[k] \cdot e[k]=\cos \left(\omega_{2} t\right) \cdot\left\{\cos \left(\omega_{1} t\right)+\cos \left(\omega_{2} t\right)+\cos \left(\omega_{3} t\right)\right\} \\
& F[\mu[k] \cdot e[k]]=\frac{1}{2} \pi\left[\delta\left(\omega+\omega_{1}-\omega_{2}\right)+\delta\left(\omega-\omega_{1}-\omega_{2}\right)\right]+ \\
& \frac{1}{2} \pi\left[\delta\left(\omega-\omega_{3}+\omega_{2}\right)+\delta\left(\omega-\omega_{3}-\omega_{2}\right)\right]+\frac{1}{2} \pi\left[\delta\left(\omega-2 \omega_{2}\right)+\delta(\omega)\right]
\end{aligned}
$$

F here means Fourier Transform and $\delta$ is Dirac Function. From (18) and (19), when $k \geq 2$, then: $\mu[k] \cdot(\mu[k] \cdot e[k])=\cos \left(\omega_{2} t\right) \cdot \cos \left(\omega_{2} t\right) \cdot\left\{\cos \left(\omega_{1} t\right)+\cos \left(\omega_{2} t\right)+\cos \left(\omega_{3} t\right)\right\}$

$$
\begin{aligned}
& F[\mu[k] \cdot(\mu[k] \cdot e[k]])=\frac{1}{4} \pi\left[\delta\left(\omega+\omega_{1}-2 \omega_{2}\right)+\delta\left(\omega-\omega_{1}-2 \omega_{2}\right)+2 \delta\left(\omega-\omega_{1}\right)\right]+\frac{1}{4} \pi[\delta(\omega+ \\
& \left.\left.\omega_{3}-2 \omega_{2}\right)+\delta\left(\omega-\omega_{3}-2 \omega_{2}\right)+2 \delta\left(\omega-\omega_{3}\right)\right]+\frac{1}{2} \pi \delta(\omega)+\frac{1}{4} \pi\left[\delta\left(\omega-\omega_{2}\right)+\delta\left(\omega-3 \omega_{2}\right)\right]
\end{aligned}
$$

Through formula(19) and (21), error signal includes other frequency spectrum components, for example: $\omega_{j}=\omega_{2} \pm \omega_{1}, \omega_{3} \pm \omega_{2}, 2 \omega_{2}, 3 \omega_{2}, 2 \omega_{2} \pm \omega_{1}, \omega_{3} \pm 2 \omega_{2}$.

Therefore the analysis above shows that this parallel structure calculation can induce other irrelevant frequency components in final error signal.

To sum up, error signal has direct effect to the stability and induction components of parallel structure multi-frequency Fx-LMS calculation. Making single error signal be feedback signal of all paths will lead to inter-connect of convergence coefficient in each path, which will influence the stability of the system and induce other frequency components[10]. Therefore, in order to achieve better integral control effect, this control multi-frequency vibration calculation must be modified.

\section{Parallel Structure Multi-frequency Fx-LMS Calculation of Signal Filter with Errors}

Figure 3 is the diagram of parallel structure multi-frequency Fx-LMS calculation of signal filter with errors. Compared with Figure 2, this calculation adds $M$ digital band-pass filter for error signal frequency separation. 


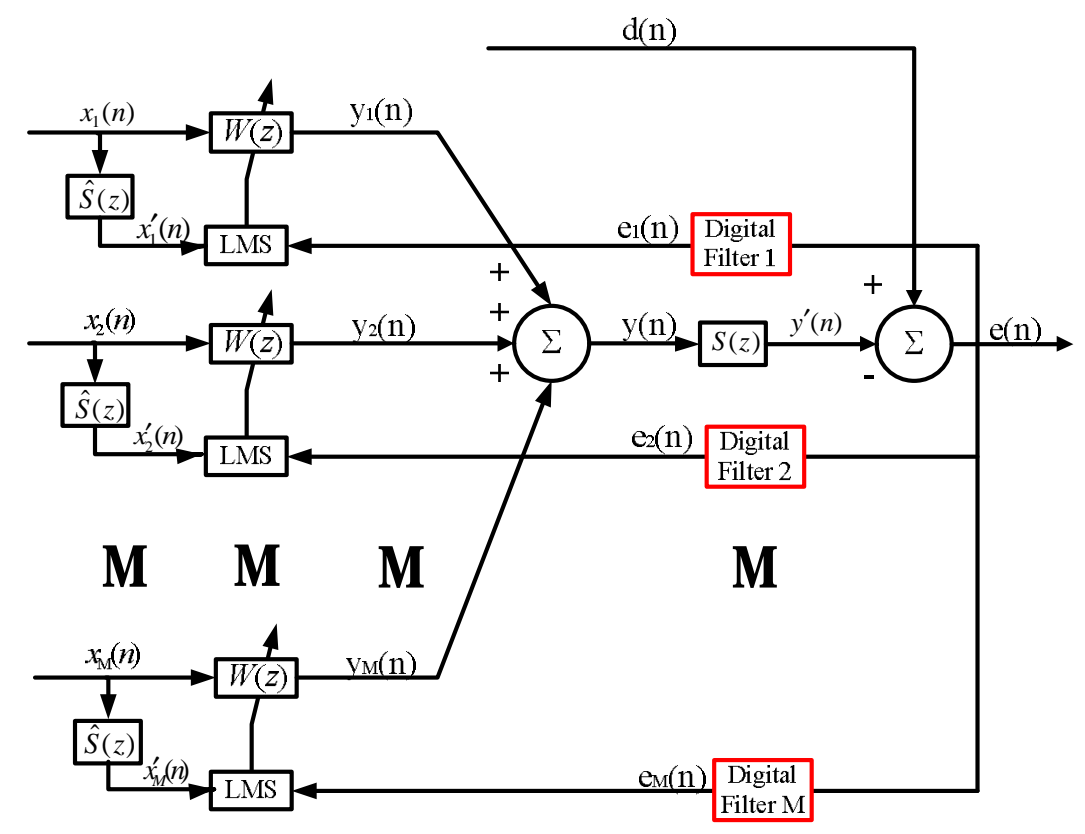

Fig. 3 Diagram of Parallel Structure Calculation of Signal Filter with Errors The iterative equation of this calculation is:

$$
w_{m}(n+1)=w_{m}(n)+\mu x_{m}^{\prime}(n) e_{m}(n) \quad, \quad m=1,2, \mathrm{~K}, M
$$

and

$$
e_{m}(n)=A_{e, m}(n) \sin \left\lfloor\omega_{m} n+\phi_{e, m}(n)\right\rfloor, m=1,2, \mathrm{~K}, M
$$

are the single frequency error signals of No.m path.

The control calculation aiming at $k \Omega$ and $2 k \Omega$ ' s components is designed after comprehensive consideration of helicopter' $\mathrm{s}$ actual vibration damping demand and complexity of calculation.

Single-input and single-output. As Figure 4 is shown, reference signal consists of the first two order frequency $x_{1}(n)$ and $x_{2}(n)$ whose vibration proportions of helicopter are greatest. $p(n)$ is primary path and $s(n)$ is error transfer function in error path. The digital model ${ }^{\hat{s}(n)}$ must be put before reference signal and adjustment filter weight coefficient. When ${ }^{w(n)}$ is $p(n)$, s optimal estimation, $y(n)$ can reappear $^{d(n)}$ better, which minimize error signal ${ }^{e(n)}$.The induction harmonic wave components can be eliminated by adding digital filter for error signal frequency separation.

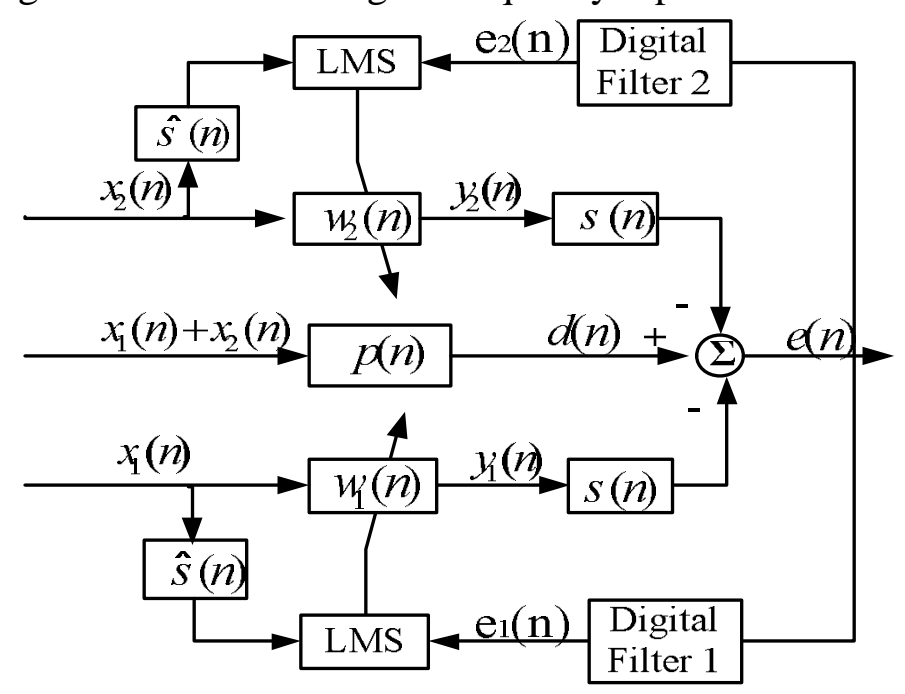

Fig. 4 Diagram of Single-input and Single-output Dual-frequency Control System 
Four-input and two-output. The dual-frequency four-input and two-output system has four main paths and eight error paths, which is shown as Figure 5.

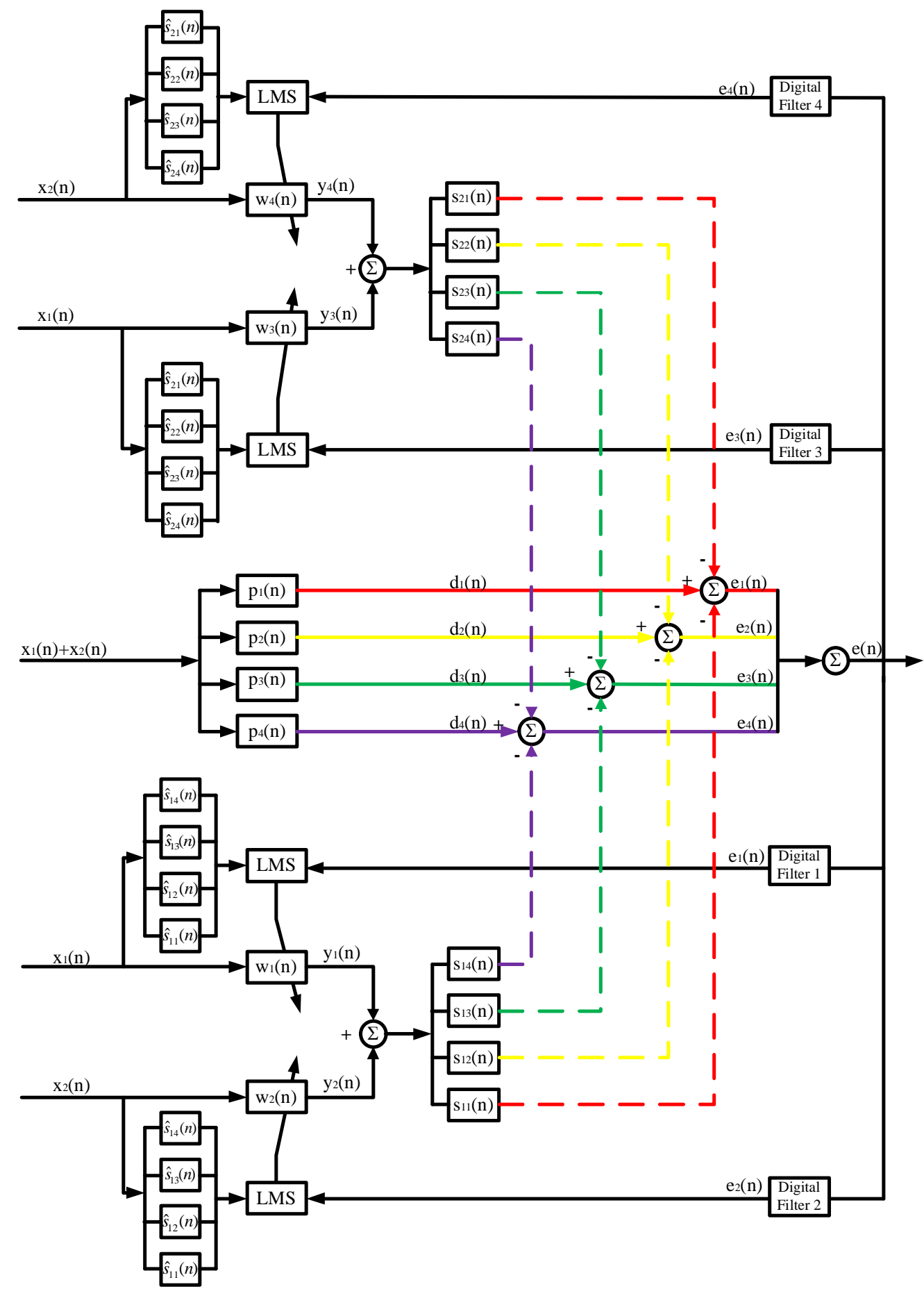

Fig. 5 Diagram of Four-input and Two-output Dual-frequency Control System

\section{Conclusion}

The modified parallel structure multi-frequency Fx-LMS calculation of signal filter with errors is carried out for the problems of stability and induction spectrum existed in traditional parallel structure multi-frequency Fx-LMS calculation. And the control calculation of $k \Omega$ and $2 k \Omega$ ' s components is 
designed after comprehensive consideration of helicopter' s actual vibration damping demand and complexity of calculation to improve the stability and control effect of helicopter' s ACSR system.

\section{References}

[1] Lu Y, Gu Z Q, Ling A M. Flight test of active control of structure response for helicopter. Journal of Vibration Engineering, 2012, 23(1): 24-29. (in Chinese) .

[2]Simon Haykin. Adaptive filter principle(fourth edition).Beijing: Electronic Industry Press, 2010.

[3] Zhou L B, Yang T J, Brennan M J, Liu Z G. Synchronous phasing Control of Floating Raft and Radiation. Harbin Engineering University, 2014,35(1):1-8.

[4] Paulo S. R. Diniz. Adaptive filter algorithm and implementation (second edition). Beijing: Electronic Industry Press, 2004.

[5] F.C. Yang, An Analysis of Narrowband Active Noise Control Systems Using Parallel Adaptive Notch Filters, MS Thesis, Northern Illinois University, May 2007.

[6] F.C. Yang, A. Gupta, and S.M. Kuo, Analysis of narrowband active noise and vibration control systems using parallel adaptive notch filters.

[7] S.M. Kuo and A.B. Puvvala, " Effects of frequency separation in periodic active noise control systems," IEEE Trans. Audio, Speech, and Language Processing, vol. 14,no. 5, pp. 1857-1866, 2006.

[8] S.M. Kuo, M. Tahernezhadi, and W. Hao," Convergence analysis of narrowband active noise control systems," IEEE Trans. on Circuits and Systems, vol. 46, no 2, pp. 220-223,Feb. 1999.

[9] Byeongil Kim, Gregory N Washington and Rajendra Singh.Control of incommensurate sinusoids using enhanced adaptive filtering algorithm based on sliding. Journal of Vibration and Control published online 8 May 2012.0(0) 1-16

[10] A. B. Puvvala, Effects of frequency separation on rate of convergence in multiple-frequency periodic noise control systems,MS thesis, Northern Illinois University, December 2004. 